\title{
Hemiplegic Migraine Presenting with Prolonged Somnolence: A Case Report
}

\author{
Christian Saleh $^{a} \quad$ Geneviève Pierquin $^{b, c} \quad$ Stefan Beyenburg ${ }^{a}$ \\ ${ }^{a}$ Department of Neurology, Centre Hospitalier de Luxembourg, Luxembourg, Luxembourg; \\ ${ }^{b}$ Génétique et Cytogénétique (T3-1), Centre Hospitalier Universitaire de Liège (C.H.U.), \\ Sart Tilman, Liège, Belgium; ${ }^{C}$ Department of Pediatrics, Centre Hospitalier de Luxembourg, \\ Luxembourg, Luxembourg
}

\section{Keywords}

Migraine $\cdot$ Hemiplegia $\cdot$ Somnolence

\begin{abstract}
Hemiplegic migraine is a rare and complex disease, characterized by migraine with a reversible motor aura. Hemiplegic migraine can be easily misdiagnosed at its first presentation with an atypical severe form of migraine, a stroke, multiple sclerosis, metabolic disorders, conversion disorder or an epilepsy. We present the case of a young 24-year-old male patient, who since the age of 4 years had been having multiple episodes of migraine associated with hemiparesis, paraesthesia, prolonged somnolence, aphasia and confusion. We review the literature and discuss important diagnostic findings in hemiplegic migraine to help establishing a prompt diagnosis.

(C) 2016 The Author(s)

Published by S. Karger AG, Basel
\end{abstract}

\section{Introduction}

Migraine is a common health condition, with an estimated prevalence of $6 \%$ among men and $16 \%$ among women [1]. However, prompt diagnosis can be difficult given the multiple and rare forms of migraine; in these cases, treatment is frequently delayed at the expenses of the patient. A rare form of migraine is hemiplegic migraine (HM), an autosomal dominant 
type of migraine typically presenting with a reversible not isolated motor aura (hemiplegia) associated with other neurological symptoms, such as seizure and coma [2, 3]. HM is differentiated into two main clinical subgroups, i.e. familial hemiplegic migraine (FHM) and sporadic hemiplegic migraine. Both have similar clinical symptoms, except the latter is without familiarity [3]. The prevalence of FHM is low $(0.005 \%)$ [4]. FHM is a genetically heterogeneous condition. Mutations in four different genes, each characterizing the four familial forms (CACNA1A/FHM1, ATP1A2/FHM2, SCN1A/FHM3, PRRT2/FHM4) were identified; these genes are involved in encoding ion transporter proteins $[5,6]$. These mutated genes are the cause of an altered neuronal excitability and reduced threshold value for cortical spreading depression (CSD), a transient slow wave of profound neuronal and glial depolarization, which spreads over the brain hemispheres or in certain cases affects the brain stem being responsible for the brain stem symptoms in FHM; CSD is also supposed to be responsible for the migrainous aura in HM $[4,7]$.

\section{Case Presentation}

A 24-year-old male presented with multiple episodes of migraine associated with hemiparesis, paraesthesia, prolonged somnolence, aphasia and confusion, which he had been having since the age of 4 years. He did not report visual disturbances or dysarthria. Each episode was characterized by a progressive reduction of symptoms and a full recovery. He was an only child. His father presented with some episodes of migraine, which, however, were never associated with other symptoms. Also, his grandmother (of paternal origin) had uncomplicated episodes of migraine. His family history was otherwise unremarkable. The patient had 2 episodes of migraine per week. The patient was extremely somnolent after these episodes (up to 24-48h). Several admissions to the intensive therapy unit were necessary. Given the 20-year history of stereotyped episodes, migraine with aura and epilepsy needed to be considered foremost. Repeated brain imaging (including also MRI angiography) were normal. Routine laboratory studies were within normal limits, anti-limbic encephalitis and NMDA-receptor antibody encephalitis were negative, and lumbar puncture was unremarkable. His electroencephalography (EEG) recording (fig. 1) showed significant changes, for his age, in form of an atypical diffuse slowing (within the delta/theta range) particularly over the left frontoparietal region corresponding to the 3-day- lasting period of somnolence. No epileptiform alterations were present. Analgesics and lamotrigine $50 \mathrm{mg}$ twice daily, subsequently increased to lamotrigine $100 \mathrm{mg}$ twice daily, were prescribed. The patient remained complain-free for several years with normalized EEG at follow-up visits (fig. 2) until he presented again with a migraine attack with hemiparesis. Lamotrigine was reduced progressively and replaced by increasing doses of topiramate (since then the patient is free of symptoms). Despite the negative family history for $\mathrm{HM}$, we conducted genetic testing. The patient had the mutation c.2936C $>\mathrm{T}$ in the gene ATP1A2. This mutation is due to a substitution of a proline to a leucine in the codon 979 (p.Pro979Leu). This mutation is known; his father did not have the mutation. Paternity testing was not done.

\section{Discussion}

$\mathrm{HM}$ is a rare and complex disease characterized by migraine with a reversible motor aura lasting from hours to days [8]. Headache presents in $95 \%$ of patients affected by HM; the 
headache (bi- or unilateral, ipsi- or contralateral to the motor weakness) can start before or after the aura [3]. HM is differentiated into FHM and sporadic HM, according to the presence or absence of familiarity [3]. HM can be easily misdiagnosed at its first presentation as an atypical severe form of migraine, a stroke, multiple sclerosis, metabolic/toxic disorders, conversion disorder or an epilepsy (Jacksonian march, Todd's paralysis) [4, 9]. The clinical diagnosis is also rendered difficult, as migraine, epilepsy and HM share not solely clinical, but also pathophysiological similarities. It is supposed that migraine attacks, epilepsy and HM might be due to neuronal cortical hyperexcitability [2, 10, 11]. Vanmolkot et al. [11] showed that approximately $75 \%$ of HM families have a mutation in CACNA1A (in FHM1) and that some CACNA1A mutations may also cause epilepsy. Penetrance of epilepsy can range between 30 and $60 \%$ in some FHM2 families [3]. These findings may explain why antiepileptic medication, such as topiramate and valproate, which suppress CSD, can be equally beneficial in the treatment of HM [10].

Over the last 10 years, there have been several reports on this disease. Weng et al. [4] reported the case of a 24-year-old male student who presented with general weakness that subsequently shifted to the right side associated with throbbing headache, right-sided hemianesthesia, hemianopia and facial palsy. Brain imaging (CT) was normal. The diagnosis of FHM was made based on similar previous attacks and a positive family history. Acetaminophen and flunarizine were prescribed during hospitalization and, for prophylaxis, verapamil. The symptoms subsided gradually without sequelae. Genetic testing was not performed. Merwick et al. [6] reported the case of a 32-year-old woman with known FHM (ATP1A2 mutation) who presented in an acute confusional state after she had suffered an HM attack and was suspected initially to have an infectious encephalitis. Her EEG showed (similar to our patient) slowing of alpha rhythm and continuous rhythmical delta-theta activity in the left hemisphere. She recovered $48 \mathrm{~h}$ after the episode with a normalization of her EEG.

Ambrosini et al. [7] described the case of a 15-year-old boy with recurrent attacks starting with an aura characterized by visual symptoms, unilateral paraesthesias, which lasted less than $15 \mathrm{~min}$, followed by unilateral pulsating headache with a duration of approximately $6 \mathrm{~h}$. Interictal neurologic examination, brain imaging (MRI) and EEG were reported to be normal. Family history was remarkable for similar episodes presenting in the patient's father and uncle. Also, the patient's cousin and mother were affected by migraine, but without aura. Genetic testing was positive for ATP1A2 mutation in the patient. A diagnosis of basilar migraine was made, but the authors underlined that basilar migraine and FHM are 'linked not only on a clinical basis, but also on a pathogenetic one'. In 2014, Tashiro et al. [12] published a case on a 38-year-old man with recurrent migraine attacks with aura, sometimes associated with hemiparesis. He had suffered from these attacks since his teenage years. Family history revealed that his mother suffered equally from migraines with hemiparesis. Based on the patient and family history findings, a clinical diagnosis of FHM was made. Subsequently, genetic testing showed that the patient and his mother had a mutation in the CACNA1A gene. Sodium valproate was prescribed, and the patient remained free of symptoms.

Diagnosis of FHM can be delayed because obtaining a detailed description of the clinical course, spanning years that included migraine episodes associated with a motor deficit [3], in the emergency department can be difficult; even during follow-up visits, patients or family members can miss to recall that some headache episodes occurred with motor deficits [3]. Russel and Ducros [3] reported that alterations of consciousness (ranging from confusion to somnolence to coma) are 'frequently, but not always associated with motor deficits'. Therefore, in a patient presenting for the first time with recurrent headache and prolonged som- 
nolence, a wide range of differential diagnoses need to be considered. Multiple conditions that can present with prolonged somnolence with EEG slowing need to be taken into consideration, such as space-occupying lesions (e.g. tumor of the pineal gland, hypothalamus), meningitis, encephalitis, a metabolic encephalopathy, drug intoxication, hypoxic states, multiple sclerosis, intracranial hemorrhage (especially when related to trauma, as in our patient) and an internal carotid artery thrombosis (to be considered by the association between hemiplegia and slow-wave EEG activity). An exhaustive diagnostic workup including brain imaging, a lumbar puncture, EEG and blood testing are critical.

Further, the diagnosis of FHM in our patient was made more difficult as there was no family history for HM; a similar case was described in the literature. Ohmura et al. [13] reported a case of a patient with sporadic hemiplegic migraine, who nevertheless presented with a CACNA1A mutation.

An important diagnostic finding in HM helping to differentiate classical migraine from HM is that the motor aura in HM is never isolated, but associated with sensory, language or visual symptoms [3]. Our patient presented with recurrent headaches and reversible motor deficits, which were associated with aphasia, prolonged somnolence and confusion. A further important differentiation factor between HM and classical migraine with aura is that minor head trauma can trigger HM [3], whereas minor head trauma is not a triggering factor of classical migraine with aura. A careful anamnesis showed that our patient had indeed suffered on three occasions a hemiparesis after a fall.

\section{Conclusion}

Migraine, albeit transitory, is a highly disabling condition that can present in multiple forms. Correct diagnosis is critical to initiate prompt treatment. A rare form of migraine is HM. Within the plethora of clinical presentations of HM, HM should be considered when the migraine aura is not isolated and associated with other symptoms. Also, multiple episodes of migraine associated with recurrent reversible encephalopathy (triggered by minor head trauma), should raise the suspicion of HM and prompt further diagnostic workup, which should always include genetic testing. Prompt diagnosis in the emergency setting is difficult as patients can present with language difficulties and the history taken is seriously hampered. Detailed family history and third-party anamnesis are critical in establishing the diagnosis. As HM shows a high phenotypic variability, clinicians should always keep a high clinical suspicion of HM in every unusual presentation of migraine.

\section{Statement of Ethics}

This material has not been published in whole or in part elsewhere; the manuscript is not currently being considered for publication in another journal; all authors have been personally and actively involved in substantive work leading to the manuscript, and will hold themselves responsible for its content. 


\section{Disclosure Statement}

The authors have no conflict of interest.

\section{References}

1 Smitherman TA, Burch R, Sheikh H, Loder E: The prevalence, impact, and treatment of migraine and severe headaches in the United States: a review of statistics from national surveillance studies. Headache 2013;53:427-436.

2 Deprez L, Weckhuysen S, Peeters K, Deconinck T, Claeys KG, Claes LR, et al: Epilepsy as part of the phenotype associated with ATP1A2 mutations. Epilepsia 2008;49:500-508.

3 Russell MB, Ducros A: Sporadic and familial hemiplegic migraine: pathophysiological mechanisms, clinical characteristics, diagnosis, and management. Lancet Neurol 2011;10:457-470.

4 Weng TH, Chiu WT, Afilalo M, Choy CS, Tselios C, Yip PK, et al: A young man presenting with acute encephalopathy, hemiparesis, and headache. J Emerg Med 2012;43:258-262.

$\checkmark 5$ Ducros A: Familial hemiplegic migraine: a model for the genetic studies of migraine. Cephalalgia 2014;34:1035-1037.

-6 Merwick A, Fernandez D, McNamara B, Harrington H: Acute encephalopathy in familial hemiplegic migraine with ATP1A2 mutation. BMJ Case Rep 2013, DOI: 10.1136/bcr-2013-009750.

7 Ambrosini A, D’Onofrio M, Grieco GS, Di Mambro A, Montagna G, Fortini D, et al: Familial basilar migraine associated with a new mutation in the ATP1A2 gene. Neurology 2005;65:1826-1828.

8 Gelfand AA, Fullerton HJ, Goadsby PJ: Child neurology: migraine with aura in children. Neurology 2010;75:e16-e19.

9 Kazemi H, Speckmann EJ, Gorji A: Familial hemiplegic migraine and spreading depression. Iran J Child Neurol 2014;8:6-11.

10 Costa C, Tozzi A, Rainero I, Cupini LM, Calabresi P, Ayata C, et al: Cortical spreading depression as a target for anti-migraine agents. J Headache Pain 2013;14:62.

11 Vanmolkot KR, Kors EE, Hottenga JJ, Terwindt GM, Haan J, Hoefnagels WA, et al: Novel mutations in the $\mathrm{Na}+, \mathrm{K}+-\mathrm{ATP}$ ase pump gene ATP1A2 associated with familial hemiplegic migraine and benign familial infantile convulsions. Ann Neurol 2003;54:360-366.

-12 Tashiro Y, Yamazaki T, Nagamine S, Mizuno Y, Yoshiki A, Okamoto K: Repeated encephalopathy and hemicerebral atrophy in a patient with familial hemiplegic migraine type 1. Intern Med 2014;53:22452250.

13 Ohmura K, Suzuki Y, Saito Y, Wada T, Goto M, Seto S: Sporadic hemiplegic migraine presenting as acute encephalopathy. Brain Dev 2012;34:691-695. 


\section{Case Reports in Neurology}

\begin{tabular}{l|l}
\hline Case Rep Neurol 2016;8:204-210 \\
\hline DOI: $10.1159 / 000448473$ & $\begin{array}{l}\text { (c) 2016 The Author(s). Published by S. Karger AG, Basel } \\
\text { www.karger.com/crn }\end{array}$ \\
\hline
\end{tabular}

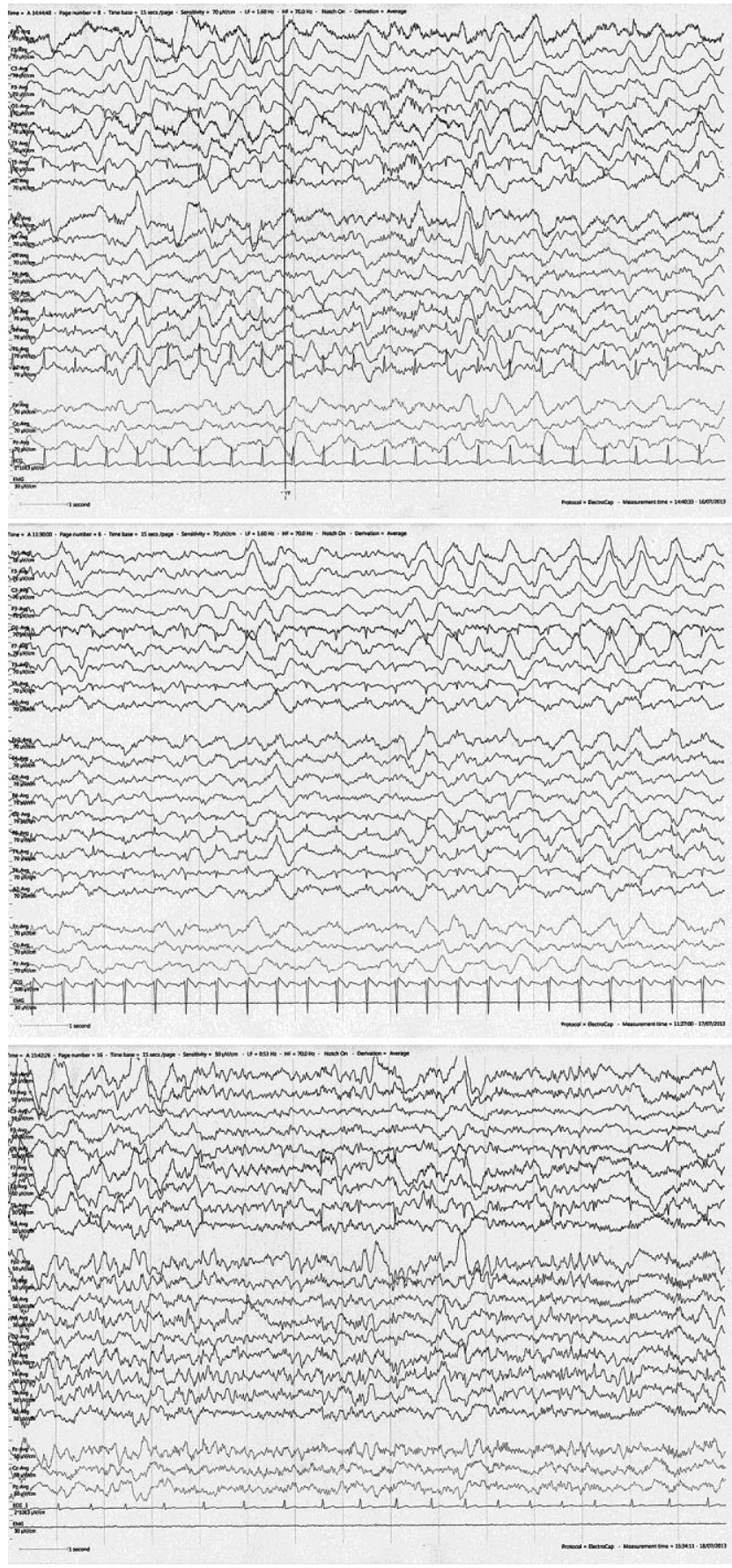

Fig. 1. The EEG trace is showing a postictal diffuse slowing in the delta-theta activity particularly over the left frontoparietal region for the course of 3 days (upper row, day 1; middle row, day 2; lower row, day 3 ). 


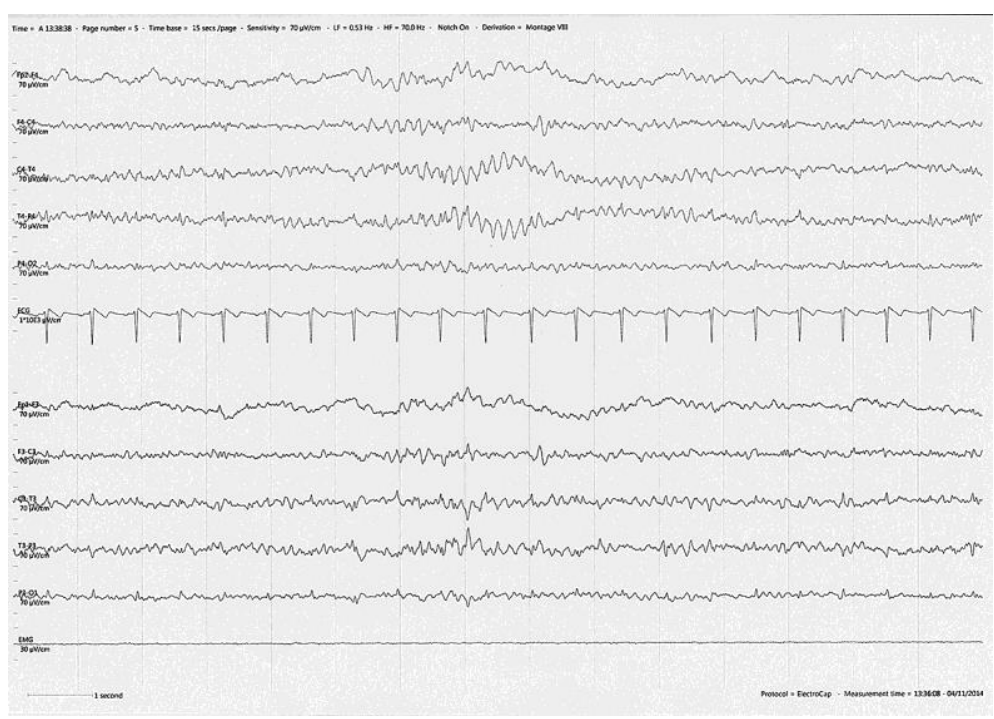

Fig. 2. The EEG at follow-up is showing a normalized trace with a temporo-occipital prominent alpha activity. 